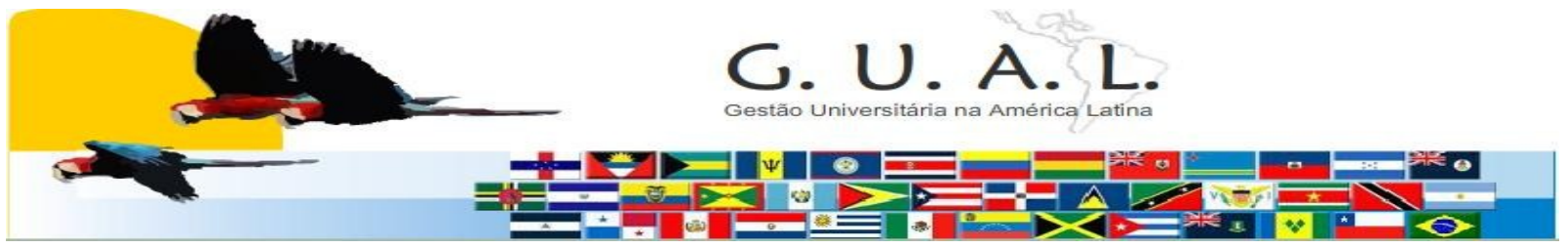

ISSN 1983-4535

\title{
CONECTIVIDADE NA CONSTRUÇÃO DE CONHECIMENTOS - ADEQUAÇÃO DA GRADE CURRICULAR NO CURSO DE ENGENHARIA DE MATERIAIS
}

\author{
Scheyla Kuester, Mestre \\ Universidade Federal de Santa Catarina - UFSC \\ scheylakuester@gmail.com
}

Daphiny Cristina Vicente Pottmaier, Doutora

Universidade Federal de Santa Catarina - UFSC daphiny@gmail.com

\author{
Andréia de Bem Machado, Mestre \\ Universidade Federal de Santa Catarina - UFSC \\ andreiadebem@gmail.com
}

Orestes Estevam Alarcon, Doutor

Universidade Federal de Santa Catarina - UFSC

orestes@emc.ufsc.br

\section{RESUMO}

A elaboração de uma grade curricular adequada pode ser feita partindo do princípio de que a construção de conhecimentos se dá por um processo de conexão entre saberes distintos em níveis crescentes de complexidade. Partindo do que já se sabe, novos conhecimentos e habilidades podem ser continuamente construídos. No ensino universitário a disposição das disciplinas na grade curricular parecem seguir certa linearidade, porém algumas vezes não ha conectividade entre seus conteúdos. Com base nisto, este artigo buscou levantar a questão da adequação curricular na construção de conhecimentos dentro do curso de Engenharia de Materiais da Universidade Federal de Santa Catarina. Para isso, foram elaborados e aplicados questionários de pesquisa de opinião e de verificação de conhecimentos prévios com os alunos de uma disciplina especifica do curso. De um modo geral, os dados apontam uma deficiência de conhecimentos prévios elementares por parte dos alunos, o que acaba por resultar em altos índices de reprovação nesta disciplina.

Palavras-chave: Aprendizagem gerida. Conhecimento significativo. Adequação curricular. 


\section{CONECTIVIDADE NA CONSTRUÇÃO DE CONHECIMENTOS - ADEQUAÇÃO DA GRADE CURRICULAR NO CURSO DE ENGENHARIA DE MATERIAIS}

\section{INTRODUÇÃO}

O fator mais importante que influencia o aprendizado é aquilo que o aprendiz já sabe. Determine e ensine de acordo com isso. (AUSUBEL, 1968, p.31)

No tratar do processo ensino-aprendizagem universitário, assim como no escolar, há que se considerar que o foco é, ou deveria ser, sempre o aluno. Ausubel (1968), em sua teoria da Aprendizagem Significativa traz a idéia de ancoragem de conhecimentos, onde uma nova informação ancora-se por meio de conhecimentos já existentes na estrutura cognitiva de quem aprende. Estes conhecimentos prévios são definidos pelo autor como "conectores".

A teoria ausubiana define como aprendizagem mecânica o processo no qual o sujeito simplesmente repete o que aprendeu, sem ter assimilado o mesmo, neste processo não houve aprendizagem significativa e não foram considerados os conhecimentos prévios dos aprendizes.

Porém, a aprendizagem mecânica não deve ser encarada como vilã, pois no princípio, quando ainda não há conhecimentos relacionáveis, ela é inevitável. Sua teoria traz a idéia de aprendizagem contínua, então a aprendizagem mecânica antecede a aprendizagem significativa objetivando as futuras ancoragens. A partir do momento em que o aluno consegue começar a fazer conexões e a assimilar conceitos, o conhecimento será ancorado e a aprendizagem será significativa. Quanto mais significativa for a aprendizagem, mais estruturada será o conhecimento do aprendiz (BRUNER, 1960), e este terá maiores capacidades para ancorar novos conhecimentos ou conhecimentos mais complexos.

No tratar do ensino universitário há que se considerar também a fase de vida em que se encontram os indivíduos ingressos e egressos. Segundo Piaget (1978), a adolescência caracteriza-se como o momento em que o indivíduo já apresenta raciocínio lógico reflexivo e a fase adulta como o momento de aprimorar as habilidades cognitivas direcionando-as ao mundo de trabalho. Neste contexto, o universo acadêmico engloba a conjuntura de transformação (PADUA, 2009).

No processo de ensino universitário as disciplinas parecem estar dispostas na grade curricular seguindo uma certa linearidade de conteúdos, porém algumas vezes os mesmos não fazem conexão entre si. Não há conectividade. Para Hartley (1998) a aprendizagem resulta 


\section{CONECTIVIDADE NA CONSTRUÇÃO DE CONHECIMENTOS - ADEQUAÇÃO DA GRADE CURRICULAR NO CURSO DE ENGENHARIA DE MATERIAIS}

das interferências, expectativas, e em fazer conexões. Ao invés de adquirir hábitos, os aprendizes adquirem planos e estratégias, para isso um conhecimento prévio é importante.

Entende-se assim que o processo não pode ser uma adição de conteúdos e sim um contínuo de assimilações, ensinar não é transferir conhecimento (FREIRE, 2003). Exemplificando, para compreender um conteúdo de estruturas de materiais na engenharia não basta conhecer a existência dos átomos e sua ligações. Então, não pode-se pensar que simplesmente acrescentar uma disciplina conceitual de química antes da disciplina sobre estruturas cristalinas será suficiente. É preciso que se faça entender o que são estes átomos, o que eles representam no meio em que se encontram, quais suas funções e porque se dispõem de um determinado modo e não de outro. Ou seja, é preciso que se criem pontes para possíveis conexões que possam resultar na assimilação dos conhecimentos. Ao considerar este caso, percebe-se que é preciso um estudo mais abrangente para perceber quais serão as disciplinas que fornecerão os conhecimentos prévios para anteceder uma certa disciplina, de modo que se torne possível sua compreensão.

No mais, a engenharia pode ser entendida como a ciência da invenção ou do engenho (LE MOIGNE, 1995). Neste contexto, o papel do engenheiro não se restringe apenas à análise, mas também às atividades de síntese e invenção. Seu produto é fruto de um processo de aprendizagem que pode ser denominado projeto de engenharia (LODER, 2009), onde projetar é o resultado de raciocinar, organizar idéias e mobilizar ações. Esses atos são possíveis através de conhecimentos acumulados sobre a natureza, as ferramentas e procedimentos. Portanto, torna-se especialmente importante aos alunos de engenharia um ambiente de aprendizagem que proporcione uma conectividade na construção dos conhecimentos.

Diante disto, o presente artigo tem por objetivo questionar a adequação curricular da disciplina Estrutura Cristalina de Sólidos no curso de Engenharia de Materiais da UFSC com base na aprendizagem significativa através da conectividade na construção dos conhecimentos.

Mais especificamente centraremos nossos esforços:

a) na identificação dos pontos fundamentais para entender a problemática da adequação curricular na construção dos conhecimentos na disciplina;

Rev. GUAL., Florianópolis, Edição especial 2011, p.195-207. 


\section{CONECTIVIDADE NA CONSTRUÇÃO DE CONHECIMENTOS - ADEQUAÇÃO DA GRADE CURRICULAR NO CURSO DE ENGENHARIA DE MATERIAIS}

b) no levantamento de informações que confirmem nossa hipótese de que o grau de complexidade e falta de conhecimento prévio requerido pela disciplina avaliada apontam para uma necessidade na mudança da estrutura curricular do curso.

A principal questão que orientara este trabalho é, portanto, a seguinte: Por que os alunos da disciplina Estrutura Cristalina de Sólidos encontram tamanha dificuldade em compreender o conteúdo da disciplina?

Esta pergunta principal será discutida a partir das seguintes perspectivas:

- perfil do curso de Engenharia de Materiais da UFSC;

- ementa da disciplina Estrutura Cristalina de Sólidos;

- avaliação dos questionários de pesquisa de opinião e de verificação dos conhecimentos prévios básicos para a compreensão da disciplina.

\section{O CURSO DE ENGENHARIA DE MATERIAIS DA UFSC}

O presente estudo foi realizado dentro do curso de graduação em Engenharia de Materiais da UFSC, criado em 1999. Este que a partir de 2001 passou a funcionar de acordo com o modelo educacional cooperativo (comumente conhecido em inglês como "co-op") em que formalmente o conhecimento e a formação do engenheiro integra estudos acadêmicos (parte disciplinar) e experiência laboral (através de estágios curriculares).

Este modelo se solidificou em 1957 pela Universidade de Waterloo no Canadá e foi estendido a diversos outras instituições, entre elas a USP que conta com três cursos desde 1989 (AULD, 1972). Alem do mais, o calendário anual do curso de engenharia de Materiais da UFSC é dividido em três períodos trimestrais compostos de quatorze semanas cada um. E a grade curricular atual é composta de nove trimestres de disciplinas nas modalidades presenciais e seis de estágios curriculares.

Apesar dos esforços por parte do corpo docente do curso que resultou em algumas modificações da estrutura curricular e conteúdo das disciplinas desde sua implementação, poucas disciplinas tem um direcionamento a aplicação pratica e imediata no exercício profissional do egresso. Este conhecimento técnico acaba por ser construído de maneira mais personalizada através da experiência individual do estudante nos estágios curriculares. No entanto, a ponte entre a fundamentação teórica e realidade profissional é deixada a cargo dos 


\section{CONECTIVIDADE NA CONSTRUÇÃO DE CONHECIMENTOS - ADEQUAÇÃO DA GRADE CURRICULAR NO CURSO DE ENGENHARIA DE MATERIAIS}

estágios curriculares e marginalizada pela parte disciplinar dentro do curso de Engenharia de Materiais.

\subsection{A DISCIPLINA ESTRUTURA CRISTALINA DE SÓLIDOS}

A engenharia de materiais tem em sua base o entendimento de materiais nos quais através da engenharia de suas estruturas se desenvolva um produto com determinadas propriedades. A correlação estrutura-propriedade é a premissa dos conhecimentos desta engenharia. Logo, para tornar possível qualquer entendimento nesta área, faz-se necessário compreender os princípios das estruturas e propriedades dos materiais.

O termo estrutura de um material pode ser compreendido como a disposição de seus componentes internos, divididos em estrutura de nível subatômico, atômico, microscópico e macroscópico. Como propriedade entende-se as características do material, dureza, flexibilidade, tenacidade, entre outras, e o comportamento do material quando submetido a estímulos externos, como forças de tração e compressão, ou quando aquecidos, por exemplo.

É neste ponto que a disciplina estrutura cristalina dos sólidos, cuja ementa é apresentada na tabela 1 , faz-se extremamente importante e fundamental. Porém, é primordial considerar que trata-se de uma disciplina elementar, mas complexa, que exige vários conhecimentos prévios para a sua compreensão.

Para compreender cristalinidade, por exemplo, precisa-se basicamente de conhecimentos claros e consistentes sobre ligações químicas, disposição atômica espacial, ordenação e solidificação. Sendo assim, coloca-se em dúvida a disposição da disciplina estrutura cristalina dos sólidos na segunda fase do curso de engenharia de materiais.

\section{Quadro 1}

Ementa da Disciplina Estrutura Cristalina de Sólidos

Programa da disciplina

1. Tipos de difração e ondas em geral.

2. DIFRAÇÃO: interferência de duas unidades e difração de $\mathrm{N}$ unidades num arranjo unidimensional. Propriedades físicas que podem ser observadas em uma dimensão.

3. Elementos de difração em três dimensões e o fator de estrutura.

4. Produção de raios-x; absorção pelo efeito fotoelétrico; absorção de raios-x.

Rev. GUAL., Florianópolis, Edição especial 2011, p.195-207. 
5. Grupos espaciais e o elemento assimétrico.

6. Difração de um pó (de cristais).

7. O difractômetro moderno de focalização tipo Bragg-Brentano.

8. Identificação de fases e a Base de Dados ICDD e "software comercial".

9. Análise quantitativa de fases.

10. Tamanho de partículas e cristais não-perfeitos.

Avaliação: O conceito final da disciplina é resultado de duas provas escritas, cada uma contando com peso de 0,4 e trabalhos com peso de 0,2 na nota final.

Carga Horária: 28 horas, 2 aulas por semana.

\section{DESENVOLVIMENTO DA PESQUISA}

Objetivando-se averiguar se a primeira fase do curso de engenharia de materiais proporciona os conhecimentos básicos para o entendimento da disciplina Estrutura Cristalina de Sólidos na segunda fase do curso realizou-se uma pesquisa de campo. A pesquisa foi realizada com alunos voluntários e dividiu-se em duas etapas, a primeira no início da disciplina (questionário 1) e a segunda na metade do trimestre letivo (questionário 2). $\mathrm{O}$ questionário 1 foi de verificação de conhecimentos, enquanto o questionário 2 dividiu-se em 2a de pesquisa de opinião e $2 \mathrm{~b}$ de verificação de conhecimentos.

O questionário 1 de verificação de conhecimentos foi realizado com alunos voluntários na primeira aula expositiva da disciplina. Este deu-se através da seguinte pergunta discursiva: "O que é um material cristalino?" onde participaram 29 alunos matriculados no curso de Engenharia de Materiais, exclusivamente.

$\mathrm{Na}$ metade do trimestre letivo, foram aplicados dois questionários de respostas objetivas (com as alternativas "sim” ou "não"). O questionário 2a foi de pesquisa de opinião onde se buscou avaliar se os alunos teriam consciência da importância, complexidade e adequação da disciplina na grade curricular. Enquanto o questionário $2 \mathrm{~b}$ foi de verificação de conhecimento onde se buscou avaliar novamente se os alunos teriam conhecimento do o que era estrutura (i), material cristalino (ii), e estado sólido (iii), conceitos pré-requisitos da disciplina. Esta segunda parte da pesquisa contou com a participação de 34 alunos voluntários 


\section{CONECTIVIDADE NA CONSTRUÇÃO DE CONHECIMENTOS - ADEQUAÇÃO DA GRADE CURRICULAR NO CURSO DE ENGENHARIA DE MATERIAIS}

e matriculados no curso de Engenharia de Materiais. Os questionários 2a e $2 b$ são apresentados a seguir nas tabelas 2 e 3 , respectivamente.

\section{Quadro 2}

Questionário 2a de pesquisa de opinião da disciplina

Você entende a importância da disciplina Estrutura Cristalina dos Sólidos no curso de Engenharia dos Materiais? ( ) Sim ( ) Não

Você acredita que no início da disciplina possuía os conhecimentos básicos para compreender a disciplina? ( ) Sim ( ) Não

Você acredita que a disciplina está disponível na fase correta do curso? ( ) Sim （ ) Não

\section{Quadro 3}

Questionário 2b sobre conhecimentos prévios para disciplina

As ligações químicas são estabelecidas entre átomos para formarem moléculas, que constituem a estrutura básica de uma substância ou composto. Estas podem ocorrer através da doação e recepção de elétrons entre os átomos (ligação iônica); através do compartilhamento de elétrons (ligação covalente); e também aquela onde os elétrons das últimas camadas dos átomos saltam e passam a se movimentar livremente entre os átomos criando uma força de atração (ligação metálica). Está afirmação é verdadeira?

( ) Sim ( ) Não

A estrutura cristalina de um sólido é a designação dada ao conjunto de propriedades que resultam da forma como estão espacialmente ordenados os átomos ou moléculas que o constituem. Esta tem as qualidades do cristal, é semelhante a ele, transparente, translúcido. Note-se que apenas os sólidos cristalinos exibem esta característica, já que ela é o resultado macroscópico da existência subjacente de uma estrutura ordenada ao nível atômico, replicada no espaço ao longo de distâncias significativas face à dimensão atômica ou molecular, o que é exclusivo dos cristais. Está afirmação é verdadeira?

( ) $\operatorname{Sim}$ ( ) Não

O estado sólido é um estado da matéria, cujas características são ter volume e forma definidos (isto é, a matéria resiste à deformação). Dentro de um sólido, os átomos ou as moléculas estão relativamente próximos, ou "rígidos". Mas isto não evita que o sólido se deforme ou comprima. Na fase sólida da matéria, os átomos têm uma ordenação espacial fixa, mas uma vez que toda a matéria tem alguma energia cinética, até os átomos do sólido mais rígido movem-se ligeiramente. Está afirmação é verdadeira? （ Sim （） Não 


\section{CONECTIVIDADE NA CONSTRUÇÃO DE CONHECIMENTOS - ADEQUAÇÃO DA GRADE CURRICULAR NO CURSO DE ENGENHARIA DE MATERIAIS}

\section{RESULTADOS E DISCUSSÕES}

$\mathrm{Na}$ primeira etapa foi aplicada uma questão para os alunos responderem de forma discursiva sobre seus conhecimentos prévios em relação à disciplina. Estes foram aplicados para avaliar o entendimento da e a percepção dos alunos quanto a importância e complexidade da disciplina. Após a coleta destas informações os dados foram compilados conforme apresentado na Figura 1.

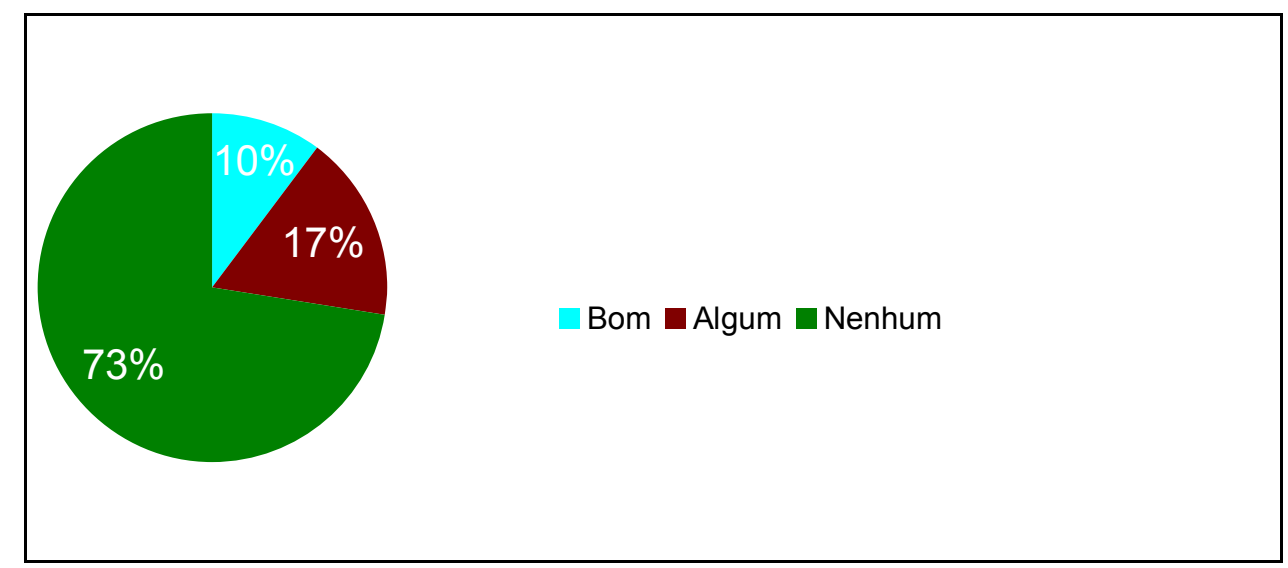

Figura 1 Resultado da pergunta discursiva "O que é um material cristalino?" para avaliação de assimilação dos conhecimentos prévios.

Na primeira aula da disciplina, antes do início da mesma, foi aplicado aos alunos o seguinte questionamento “O que é um material cristalino?”. A questão foi respondida de forma individual por escrito e entregue em seguida. As respostas foram analisadas de forma a entender se os alunos tinham um Bom (A), Algum (B) ou Nenhum (C) conhecimento prévio assimilado, considerando este um conceito básico e parte da fundamentação teórica para a compreensão dos conceitos futuros a serem abordados pela disciplina.

Para critérios de classificação foi utilizada a definição de material cristalino do livro Ciência e Engenharia dos Materiais - Uma introdução, de William D. Callister (CALLISTER, 2006). Dos 29 alunos participantes somente 10,35\% foram considerados A, 17,24\% considerados B e 72,41\% considerados C. Deste modo, evidencia-se que $89,65 \%$ dos estudantes não teriam assimilado previamente os conhecimentos para transpor este conceito básico de forma organizada.

$\mathrm{Na}$ segunda etapa foram aplicados dois questionários de respostas objetivas (com as alternativas "sim” ou "não"). O primeiro questionário buscou avaliar a visão e opinião dos 


\section{CONECTIVIDADE NA CONSTRUÇÃO DE CONHECIMENTOS - ADEQUAÇÃO DA GRADE CURRICULAR NO CURSO DE ENGENHARIA DE MATERIAIS}

alunos sobre a disciplina. O segundo questionário objetivou averiguar se os alunos tinham um conhecimento assimilado e organizado sobre o que é estrutura, material cristalino, e estado sólido, considerados conceitos básicos para a disciplina. Todos os alunos que participaram respondendo o questionário específico sobre a disciplina também responderam o questionário sobre os conhecimentos prévios da mesma.

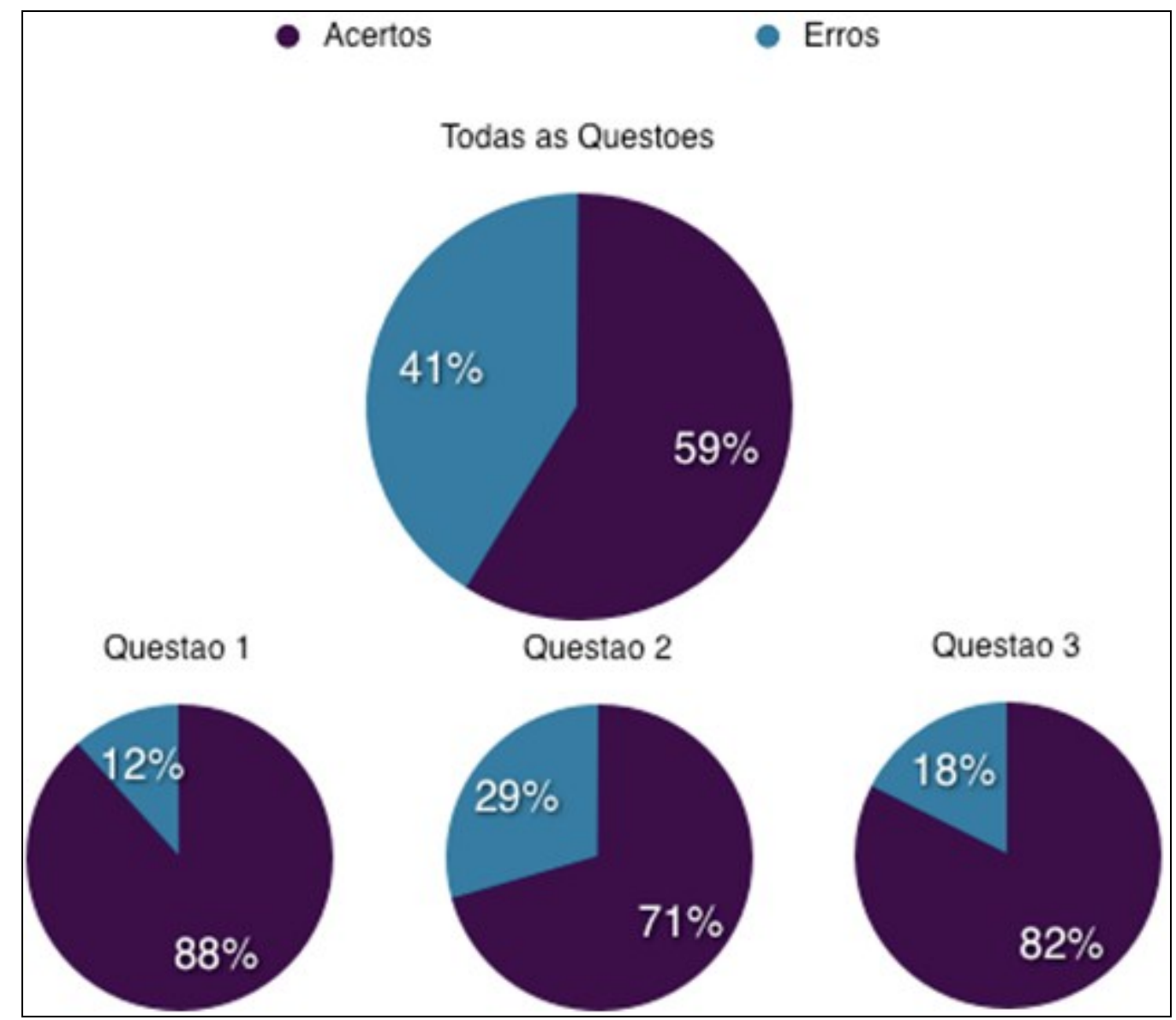

Figura 2 Resultado do segundo questionário para verificação dos conhecimentos prévios

Dos 34 alunos participantes do questionário de opinião sobre a disciplina 97,06\% responderam entender a importância da disciplina, 70,58\% acreditam que não possuíam os conhecimentos prévios para compreender a disciplina no inicio da mesma, e 88,23\% acreditam que a disciplina não está disponível na fase correta do curso.

Dos alunos participantes do questionário para verificação dos conhecimentos prévios da disciplina somente $58,82 \%$ acertaram todas as questões, sendo que destes $11,77 \%$ não responderam corretamente a questão sobre ligações químicas, 29,41\% não responderam 


\section{CONECTIVIDADE NA CONSTRUÇÃO DE CONHECIMENTOS - ADEQUAÇÃO DA GRADE CURRICULAR NO CURSO DE ENGENHARIA DE MATERIAIS}

corretamente a questão sobre estrutura cristalina, e 17,65\% não acertaram a questão sobre estado sólido. Os resultados confirmam a ma adequação da disciplina, considerando que este questionário foi respondido na sexta semana de curso, de um total de quatorze semanas letivas, e que se tratam de questões conceituais básicas necessárias à compreensão da mesma.

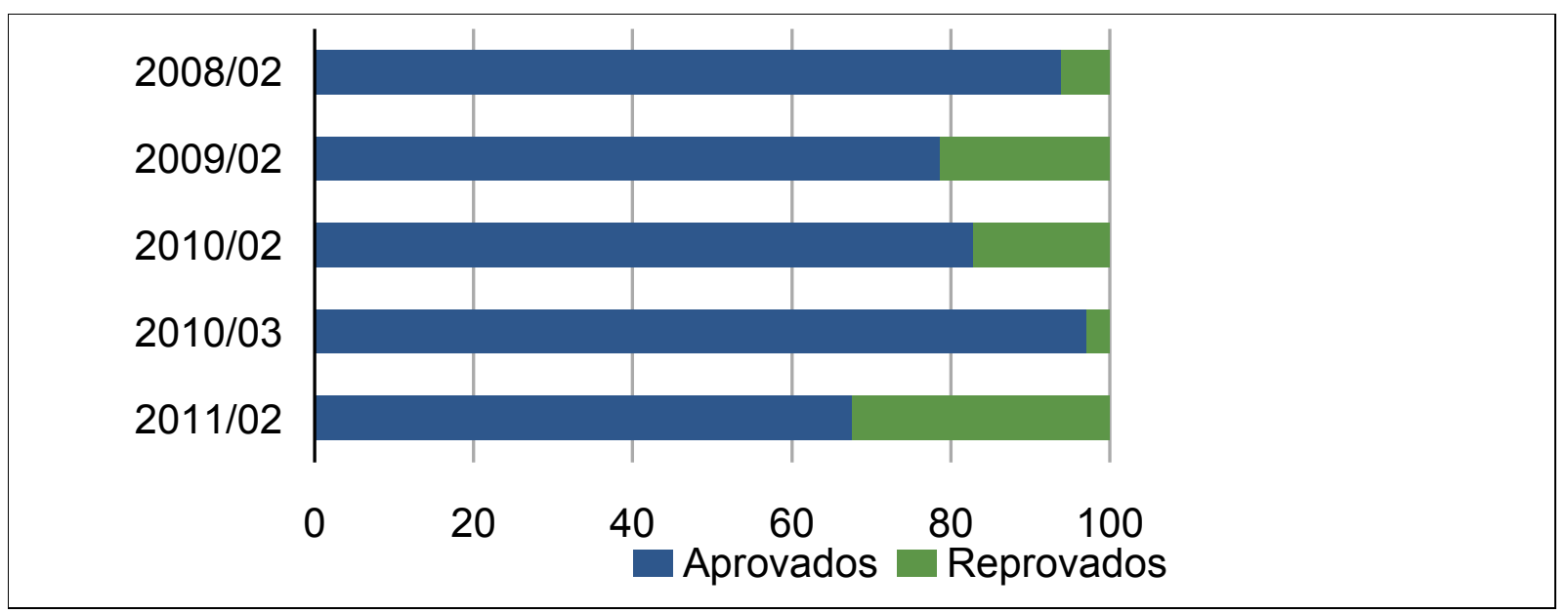

Figura 3 Relação percentual entre aprovados e reprovados na disciplina por trimestre.

Ao analisar os dados de aprovações e reprovações em cursos de engenharias, é comum observar que, em geral, as disciplinas com maior número de reprovações são as relacionadas a cálculo. Em particular, dentre os dados de reprovações do curso de engenharia de materiais da UFSC é possível notar que no caso das disciplinas que não são constituídas de grande número de cálculo, a disciplina estrutura cristalina dos sólidos apresenta elevado grau de reprovações. A média de reprovação da disciplina é de 15,91\%, levando em consideração os números apresentados na figura 3 desde sua implantação em 2008 até o primeiro semestre de 2011. Ao analisar a ementa da disciplina e considerar a pequena presença de cálculos, infere-se que o elevado grau de dificuldade por parte dos alunos é proveniente da complexidade do conteúdo da mesma.

\section{CONSIDERAÇÕES FINAIS}

O processo de ensino-aprendizagem precisa ser compreendido como ação contínua, onde os conhecimentos adquiridos anteriormente servem como pontes para conectar os novos, permitindo assim a assimilação dos mesmos para uma aprendizagem significativa.

Rev. GUAL., Florianópolis, Edição especial 2011, p.195-207. 


\section{CONECTIVIDADE NA CONSTRUÇÃO DE CONHECIMENTOS - ADEQUAÇÃO DA GRADE CURRICULAR NO CURSO DE ENGENHARIA DE MATERIAIS}

Observando a disposição das disciplinas na grade curricular do curso de engenharia de materiais da UFSC e os resultados das análises do questionamento primeiro e dos posteriores questionários, chegou-se a conclusão de que os alunos ingressantes na segunda fase do curso não possuem conhecimentos prévios suficientes para uma compreensão adequada da disciplina Estrutura Cristalina dos Sólidos.

Por se tratar de uma disciplina de difícil compreensão, que ao mesmo tempo é caracterizada por uma extrema importância, coloca-se em questão se a mesma não deveria ser redirecionada a outra fase do curso. Pois, considerando seu grau de complexidade e a falta dos conhecimentos prévios exigidos, os alunos acabam não conseguindo assimilar seu conteúdo de forma significativa.

A pesquisa apresentada visa estimular uma discussão sobre o tema conectividade da construção dos conhecimentos dentro do curso de Engenharia de Materiais da UFSC. Sugerese que seja feito um estudo abrangente das ementas das disciplinas do curso a fim de descobrir qual o melhor posicionamento para a disposição da disciplina Estrutura Cristalina dos Sólidos. Adicionalmente, aponta-se a importância de uma contínua avaliação das disciplinas dos cursos de nível superior, a fim de prover uma melhor adequação da grade curricular.

\section{AGRADECIMENTOS}

Os autores gostariam de agradecer à professora e aos alunos da disciplina Estrutura Cristalina dos Sólidos do curso de Engenharia de Materiais da UFSC que aceitaram voluntariamente participar desta pesquisa.

\section{REFERÊNCIAS BIBLIOGRÁFICAS}

AULD, Robert B. "The Cooperative Education Movement: Association of Cooperative Colleges" In: Journal of Cooperative Education 8 (5): 24-27, 1972.

AUSUBEL, David Paul. Educational Psychology: a cognitive view. New York: Holt Rinehart and Winston Inc., 1968.

BRUNER, Jerome The process of Education. Cambridge Mass.: Harvard University Press, 1960.

CALlister, William Fundamentos da Ciência e Engenharia de Materiais. Rio de Janeiro: LTC, 2006. 
FREIRE, Paulo Pedagogia da Autonomia. São Paulo: Paz e Terra, 2003.

HARTLEY, James Learning and Studying: a research perspective, London: Routledge, 1998.

LE MOIGNE, Jean-Louis. O construtivismo das epistemologias. Lisboa: Instituto Jean Piaget. 1995. 1995. , Jean-Louis. O construtivismo dos fundamentos. Lisboa: Instituto Jean Piaget.

LODER, Liane Ludwig. Engenheiro em formação: $O$ sujeito da aprendizagem e a construção do conhecimento em engenharia elétrica. Porto Alegre: UFRGS, 2009. 320f. + Anexos. Tese (Doutorado em Educação) - Programa de Pós-Graduação em Educação, Faculdade de Educação, Universidade Federal do Rio Grande do Sul, Porto Alegre, 2009.

PÁDUA, Gelson Luiz Daldegan de. A Epistemologia Genética de Jean Piaget. Revista FACEVV, Vila Velha (ES), n. 2, p. 22-35, $1^{\circ}$ Semestre de 2009.

PIAGET, Jean. A epistemologia genetica. Sabedoria e ilusoes da filosofia. Problemas de psicologia genetica.. São Paulo (SP): Abril Cultural, 1978.

, J. (1980) As formas elementares da dialética. Tradução de Fernanda Mendes Luiz. São Paulo: Casa do Psicólogo, 1996.

, Jean. Development and Learning. In LAVATTELLY, C, S. e STENDLER, F. Reading in child behavior ad development. New York: Hartcourt Brace Janovich, 1927. (Tradução: SLOMP, Paulo F. Revisão: Becker, Fernando.) 


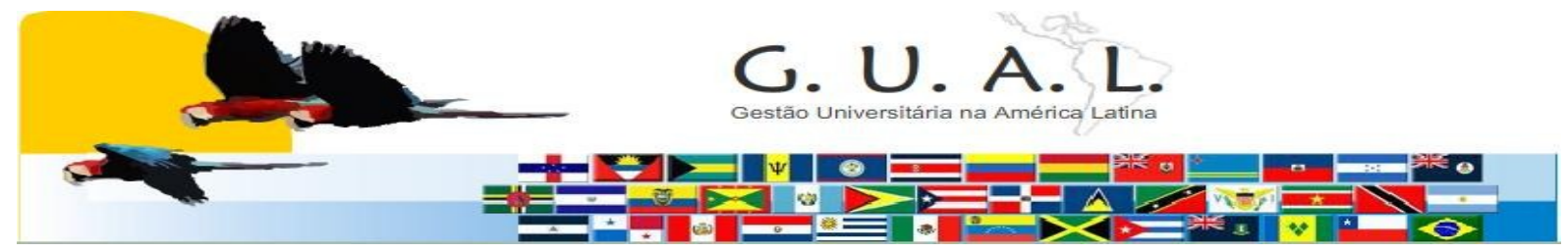

ISSN 1983-4535

\title{
CONNECTIVITY IN THE CONSTRUCTION OF KNOWLEDGE: ADEQUACY OF THE SCHOLAR CURRICULUM ON THE MATERIALS ENGINEERING COURSE
}

\author{
Scheyla Kuester, Master \\ Universidade Federal de Santa Catarina - UFSC \\ scheylakuester@gmail.com
}

Daphiny Cristina Vicente Pottmaier, Doctor

Universidade Federal de Santa Catarina - UFSC

daphiny@gmail.com

\author{
Andréia de Bem Machado, Master \\ Universidade Federal de Santa Catarina - UFSC \\ andreiadebem@gmail.com
}

Orestes Estevam Alarcon, Doctor

Universidade Federal de Santa Catarina - UFSC

orestes@emc.ufsc.br

\begin{abstract}
Scholar curriculum can be designed from a perspective that knowledge construction is made from a connection process between distinct information in progressive levels of complexity. Starting from what one knows, new understandings and abilities can be continuously constructed. In higher education disciplines in the scholar curriculum seems to be linearly arranged, however, sometimes there is no connectivity among subjects. Based on that, the present paper aims to raise the question of the curriculum adequacy in the construction of knowledge within the course of Materials Engineering of Federal University of Santa Catarina. To this end, questionnaires were developed and implemented with students in a specific discipline of the course for an opinion survey and for verification of prior knowledge. In general, the data show a deficiency of prior and elementary knowledge on the part of students, which ultimately result in high failing rates in this discipline.
\end{abstract}

Keywords: Learning. Meaningful knowledge. Curricular adequacy. 